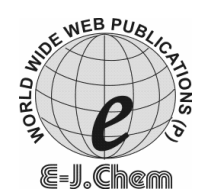

http://www.e-journals.net
ISSN: 0973-4945; CODEN ECJHAO

E-Journal of Chemistry 2010, 7(1), 281-286

\title{
A Facile Microwave Assisted One-pot Strategy for the Synthesis of Bis-hexahydroquinazolin-5(6H)-ones
}

\author{
MILAN CH. DUTTA ${ }^{\S}$, KAUSHIK CHANDA ${ }^{\S}$, \\ R. L. NONGKHLAW and JAI N. VISHWAKARMA ${ }^{\S^{*}}$ \\ ${ }^{\S}$ Organic Research Lab., Department of Chemistry, \\ St. Anthony's College, Shillong-793 001, India. \\ Department of Chemistry, \\ North-Eastern Hill University, Shillong-793 022, India. \\ jnvishwakarma@ rediffmail.com
}

Received 15 June 2009; Accepted 18 July 2009

\begin{abstract}
A facile microwave assisted one-pot synthetic protocol has been devised for the synthesis of 3,3'-(alkanediyl) bis (1-phenyl-1,2,3,4,7,8hexahydroquinazolin-5(6H)-ones (2a-c), 3,3'-(1,4-phenylene) bis (1-phenyl1,2,3,4,7,8-hexahydroquinazolin-5(6H)-one (2d),3,3'-(alkanediyl) bis (7,7dimethyl-1-phenyl-1,2,3,4,7,8-hexahydroquinazolin-5(6H)-ones (2e-g) and 3,3'-(1,4-phenylene) bis(7,7-dimethyl-1-phenyl-1,2,3,4,7,8-hexahydroquinazolin$5(6 \mathrm{H})$-one $(\mathbf{2 h})$ by the cyclocondensation of cyclic enaminones $\mathbf{1 a}, \mathbf{b}$ with diamines and formaldehyde.
\end{abstract}

Keywords: Enaminones, Quinazolinones, Diamines, Cyclocondensation.

\section{Introduction}

Fused pyrimidines are found in a broad variety of natural products ${ }^{1}$, used in medicines ${ }^{2}$, possess antimalarial activities ${ }^{3}$ and other important biological properties ${ }^{4}$. Recently, 1,2,3,4,5,6,7,8-octahydroquinazoline-2,5-dione derivatives have been reported to exhibit potent calcium antagonist activities ${ }^{5,6}$ and have also attracted considerable attention owing to their potential antibacterial activity against Staphylococcus aureus, Escherichia coli, Pseudomonas aeruginosa ${ }^{7,8}$. However, synthesis of 5-oxo-1,2,3,4,5,6,7,8-octahydroquinazolines ${ }^{9}$ is least attended to and to the best of our knowledge bis(5-oxo-1,2,3,4,5,6,7,8octahydroquinazolines) are unknown in the literature and hence their biological properties remain unexplored. Prompted by the promising biological properties of 1,2,3,4,5,6,7,8octahydroquinazolines, we have recently reported ${ }^{10}$ a facile synthetic methodology for 1aralkyl/aryl-3-alkyl/aralkyl/aryl-5-oxo-1,2,3,4,5,6,7,8-octahydroquinazolines and 1-aralkyl/aryl3-alkyl/aralkyl/aryl-7,7-dimethyl-5-oxo-1,2,3,4,5,6,7,8-octahydroquinazolines. In continuation with our efforts on the synthesis of tetrahydropyrimidines ${ }^{11-14}$, we now report herein a facile 
one pot synthetic strategy for bis-1,2,3,4,7,8-hexahydroquinazolin-5(6H)-ones in which the two quinazoline rings are linked through flexible aliphatic chains or through rigid aromatic ring (Scheme 1).<smiles>[R]C1([R])CC(=O)C=C(Nc2ccccc2)C1</smiles>

1

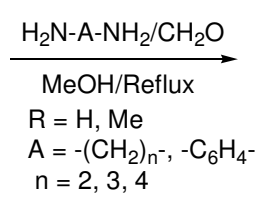

Scheme 1<smiles>[R]C1([R])CC(=O)C2=C(C1)N(c1ccccc1)CN([Y]N1CC3=C(CC([R])([R])CC3=O)N(c3ccccc3)C1)C2</smiles>

2

\section{Experimental}

Melting points were recorded by open capillary method and are uncorrected. The IR spectra were recorded on a Perkin-Elmer 983 spectrometer. ${ }^{1} \mathrm{H}$ NMR (90 MHz) spectra were recorded on Varian EM-390 spectrometer. High resolution ${ }^{1} \mathrm{H}$ NMR and ${ }^{13} \mathrm{C}$ NMR (300 $\mathrm{MHz}$ ) spectra were recorded on Bruker ACF-300 spectrometer. The chemical shifts ( $\delta \mathrm{ppm})$ and the coupling constants $(\mathrm{Hz})$ are reported in the standard fashion with reference to TMS as internal reference. FAB-mass spectra (MS) were measured on JEOL 3SX 102/DA-6000 Mass spectrometer using Argon as the FAB gas and $m$-nitrobenzylalcohol as the matrix. Elemental analyses were performed on a Vario-EL III instrument. Microwave irradiation was carried out in a domestic MW oven (Samsung CE2733G) operating at $2450 \mathrm{MHz}$. Enaminones 1a and $\mathbf{1 b}$ were synthesized by our reported procedure ${ }^{15}$.

\section{General procedure}

A mixture of diamine $(0.5 \mathrm{mmol})$ and formaldehyde $(2 \mathrm{mmol}, 40 \%$ solution $)$ in $1.5 \mathrm{~mL}$ methanol was shaken at room temperature for 5 minutes. To this a solution of enaminones 1 $(1 \mathrm{mmol})$ was added in 5-6 mL methanol and the resulting mixture was irradiated in a domestic microwave oven for specified period of time (Table 2). After the completion of the reaction (monitored by TLC), methanol was removed under reduced pressure to give a gum, which on trituration with hexane and subsequent recrystallization in appropriate solvent gave compounds $\mathbf{2 a}, \mathbf{2 e}, \mathbf{2 g}$ and $\mathbf{2 h}$. In case of compounds $\mathbf{2 b}, \mathbf{2 c}, \mathbf{2 d}$ and $\mathbf{2 f}$ the gum was chromatographed using neutral alumina and ethylacetate (eluant). Under thermal conditions these reactions took much longer time for completion giving the desired products in comparatively lower yields.

\section{3,3'-(Ethane-1,2-diyl)bis(1-phenyl-1,2,3,4,7,8-hexahydroquinazolin-5(6H)-one (2a)}

It was obtained as a pale yellow solid in $85 \%$ yield; mp $182-184{ }^{\circ} \mathrm{C}$ (EtOAc); IR (KBr): 1493, $1566 \mathrm{~cm}^{-1} ;{ }^{1} \mathrm{H}$ NMR $\left(\mathrm{CDCl}_{3}\right): \delta 1.70\left(\mathrm{~m}, 4 \mathrm{H}, 2 \mathrm{CH}_{2}\right), 2.12-2.28\left(\mathrm{t}, 4 \mathrm{H}, 2 \mathrm{CH}_{2}\right), 2.31$ $2.60\left(\mathrm{t}, 4 \mathrm{H}, 2 \mathrm{CH}_{2}\right), 2.83\left(\mathrm{~s}, 4 \mathrm{H}, 2 \mathrm{CH}_{2}\right), 3.73\left(\mathrm{~s}, 4 \mathrm{H}, 2 \mathrm{CH}_{2}\right), 4.43\left(\mathrm{~s}, 4 \mathrm{H}, 2 \mathrm{CH}_{2}\right), 7.07-7.87$ (m, 10H); MS: $\mathrm{m} / \mathrm{z} 483\left(\mathrm{MH}^{+}\right.$). Anal. Calc. for $\mathrm{C}_{30} \mathrm{H}_{34} \mathrm{~N}_{4} \mathrm{O}_{2}$ (482.27): C, 74.66; H, 7.10; N, 11.61. Found: C, 74.41; H, 7.07; N, 11.66\%.

\section{3,3'-(Propane-1,3-diyl)bis(1-phenyl-1,2,3,4,7,8-hexahydroquinazolin-5(6H)-one (2b)}

It was obtained as yellow gum in $76 \%$ yield; IR $\left(\mathrm{CCl}_{4}\right): 1493,1560 \mathrm{~cm}^{-1} ;{ }^{1} \mathrm{H}$ NMR $\left(\mathrm{CDCl}_{3}\right): \delta 1.84-1.85\left(\mathrm{~m}, 4 \mathrm{H}, 2 \mathrm{CH}_{2}\right), 1.86-1.88(\mathrm{~m}, 2 \mathrm{H}), 2.18-2.20\left(\mathrm{t}, 4 \mathrm{H}, 2 \mathrm{CH}_{2}\right), 2.32-$ $2.36\left(\mathrm{t}, 4 \mathrm{H}, 2 \mathrm{CH}_{2}\right), 2.67-2.72\left(\mathrm{t}, 4 \mathrm{H}, 2 \mathrm{CH}_{2}\right), 3.64\left(\mathrm{~s}, 4 \mathrm{H}, 2 \mathrm{CH}_{2}\right), 4.30\left(\mathrm{~s}, 4 \mathrm{H}, 2 \mathrm{CH}_{2}\right)$, 7.11-7.13 (m, 4H), 7.27-7.43 (m, 6H); ${ }^{13} \mathrm{C}$ NMR $\left(\mathrm{CDCl}_{3}\right): \delta 22.10,26.07,27.72,36.55$, 47.96, 50.95, 71.98, 104.83, 127.36, 127.50, 129.84, 143,02, 158.36, 194.94; MS: m/z, $497\left(\mathrm{MH}^{+}\right)$. Anal. Calc. for $\mathrm{C}_{31} \mathrm{H}_{36} \mathrm{~N}_{4} \mathrm{O}_{2}$ (496.28): C, 74.97; H, 7.31; N, 11.28. Found: $\mathrm{C}$, $74.66 ; \mathrm{H}, 7.33 ; \mathrm{N}, 11.24 \%$. 
3,3'-(Butane-1,4-diyl)bis(1-phenyl-1,2,3,4,7,8-hexahydroquinazolin-5(6H)-one (2c)

It was obtained as yellow gum in $77 \%$ yield; IR $\left(\mathrm{CCl}_{4}\right): 1493,1560 \mathrm{~cm}^{-1} ;{ }^{1} \mathrm{H} \mathrm{NMR}\left(\mathrm{CDCl}_{3}\right)$ : $\delta 1.58\left(\mathrm{~m}, 4 \mathrm{H}, 2 \mathrm{CH}_{2}\right), 1.85-1.88\left(\mathrm{~m}, 4 \mathrm{H}, 2 \mathrm{CH}_{2}\right), 2.21\left(\mathrm{t}, 4 \mathrm{H}, 2 \mathrm{CH}_{2}\right), 2.33\left(\mathrm{t}, 4 \mathrm{H}, 2 \mathrm{CH}_{2}\right)$, $2.62\left(\mathrm{t}, 4 \mathrm{H}, 2 \mathrm{CH}_{2}\right), 3.64\left(\mathrm{~s}, 4 \mathrm{H}, 2 \mathrm{CH}_{2}\right), 4.29$ (s, 4H, $\left.2 \mathrm{CH}_{2}\right), 7.12-7.14(\mathrm{~m}, 4 \mathrm{H}), 7.30-7.43$ $(\mathrm{m}, 6 \mathrm{H}) ;{ }^{13} \mathrm{C} \mathrm{NMR}\left(\mathrm{CDCl}_{3}\right): \delta 22.08,25.59,27.71,30.51,47.59,52.59,72.19,104.76$, 124.61, 127.37, 129.31, 143,05, 158.48, 195.03; MS: m/z, $511\left(\mathrm{MH}^{+}\right)$. Anal. Calc. for $\mathrm{C}_{32} \mathrm{H}_{38} \mathrm{~N}_{4} \mathrm{O}_{2}$ (510.29): C, 75.26; H, 7.50; N, 10.97. Found: C, 75.50; H, 7.48; N, 10.92\%.

3,3'-(1,4-Phenylene)bis(1-phenyl-1,2,3,4,7,8-hexahydroquinazolin-5(6H)-one (2d)

It was obtained as yellow solid in $79 \%$ yield; mp 191-193 ${ }^{\circ} \mathrm{C}$; IR (KBr): 1427, 1507, 1566 $\mathrm{cm}^{-1} ;{ }^{1} \mathrm{H}$ NMR $\left(\mathrm{CDCl}_{3}\right): \delta 1.70-2.06\left(\mathrm{~m}, 4 \mathrm{H}, 2 \mathrm{CH}_{2}\right), 2.10-2.30\left(\mathrm{t}, 4 \mathrm{H}, 2 \mathrm{CH}_{2}\right), 2.33-2.60(\mathrm{t}$, $\left.4 \mathrm{H}, 2 \mathrm{CH}_{2}\right), 4.56\left(\mathrm{~s}, 4 \mathrm{H}, 2 \mathrm{CH}_{2}\right), 4.99\left(\mathrm{~s}, 4 \mathrm{H}, 2 \mathrm{CH}_{2}\right), 6.96-7.93(\mathrm{~m}, 14 \mathrm{H}) ; \mathrm{MS}: \mathrm{m} / \mathrm{z}, 531$ $\left(\mathrm{MH}^{+}\right.$). Anal. Calc. for $\mathrm{C}_{34} \mathrm{H}_{34} \mathrm{~N}_{4} \mathrm{O}_{2}$ (530.27): C, 76.95; H, 6.46; N, 10.56. Found: C, 76.70; $\mathrm{H}, 6.45 ; \mathrm{N}, 10.51 \%$.

3,3'-(Ethane-1,2-diyl)bis(7,7-dimethyl-1-phenyl-1,2,3,4,7,8-hexahydroquinazolin$5(6 H)$-one $(2 e)$

It was obtained as yellow solid in $87 \%$ yield; mp $193-194{ }^{\circ} \mathrm{C}\left(\mathrm{CHCl}_{3} / \mathrm{Hexane}\right)$; IR ( $\left.\mathrm{KBr}\right)$ : 1493, 1566, $1619 \mathrm{~cm}^{-1} ;{ }^{1} \mathrm{H}$ NMR $\left(\mathrm{CDCl}_{3}\right): \delta 1.03\left(\mathrm{~s}, 12 \mathrm{H}, 4 \mathrm{CH}_{3}\right), 2.10\left(\mathrm{~s}, 4 \mathrm{H}, 2 \mathrm{CH}_{2}\right), 2.23$ $\left(\mathrm{s}, 4 \mathrm{H}, 2 \mathrm{CH}_{2}\right), 2.93\left(\mathrm{~s}, 4 \mathrm{H}, 2 \mathrm{CH}_{2}\right), 3.80\left(\mathrm{~s}, 4 \mathrm{H}, 2 \mathrm{CH}_{2}\right), 4.50\left(\mathrm{~s}, 4 \mathrm{H}, 2 \mathrm{CH}_{2}\right), 7.03-7.76(\mathrm{~m}$, $10 \mathrm{H})$; MS: $\mathrm{m} / \mathrm{z}, 539\left(\mathrm{MH}^{+}\right)$. Anal. Calc. for $\mathrm{C}_{34} \mathrm{H}_{42} \mathrm{~N}_{4} \mathrm{O}_{2}$ (538.33): C, 75.80; H, 7.86; N, 10.40. Found: C, 76.05; H, 7.88; N, 10.44\%.

3,3'-(Propane-1,3-diyl)bis(7,7-dimethyl-1-phenyl-1,2,3,4,7,8-hexahydroquinazolin$5(6 H)$-one $(2 f)$

It was obtained as yellow gum in $75 \%$ yield; IR $\left(\mathrm{CCl}_{4}\right): 1427,1493,1566, \mathrm{~cm}^{-1} ;{ }^{1} \mathrm{H}$ NMR $\left(\mathrm{CDCl}_{3}\right): \delta 0.90\left(\mathrm{~s}, 12 \mathrm{H}, 4 \mathrm{CH}_{3}\right), 1.59-1.61(\mathrm{~m}, 2 \mathrm{H}), 1.99\left(\mathrm{~s}, 4 \mathrm{H}, 2 \mathrm{CH}_{2}\right), 2.13(\mathrm{~s}, 4 \mathrm{H}, 2$ $\mathrm{CH}_{2}$ ), 2.60-2.65 (m, 4H, $\left.2 \mathrm{CH}_{2}\right) 3.58\left(\mathrm{~s}, 4 \mathrm{H}, 2 \mathrm{CH}_{2}\right), 4.24\left(\mathrm{~s}, 4 \mathrm{H}, 2 \mathrm{CH}_{2}\right), 7.01-7.08(\mathrm{~m}, 4 \mathrm{H})$, 7.25-7.41 (m, 6H); ${ }^{13} \mathrm{C}$ NMR $\left(\mathrm{CDCl}_{3}\right): \delta 24.92,27.28,27.36,31.64,39.99,46.40,48.65$, 48.96, 49.46, 70.92, 102.15, 126.38, 127.89, 129.39, 141.78, 155.62, 193.42. Anal. Calc. for $\mathrm{C}_{35} \mathrm{H}_{44} \mathrm{~N}_{4} \mathrm{O}_{2}$ (552.35): C, 76.05; H, 8.02; N, 10.15. Found: C, 76.31; H, 7.98; N, $10.11 \%$.

3,3'-(Butane-1,4-diyl)bis(7,7-dimethyl-1-phenyl-1,2,3,4,7,8-hexahydroquinazolin$5(6 H)$-one $(2 \mathrm{~g})$

It was obtained as a pale yellow solid in $90 \%$ yield; mp $168-169{ }^{\circ} \mathrm{C}(\mathrm{EtOAc})$; IR (KBr): 1440, 1566, $1613 \mathrm{~cm}^{-1} ;{ }^{1} \mathrm{H}$ NMR $\left(\mathrm{CDCl}_{3}\right): \delta 1.03\left(\mathrm{~s}, 12 \mathrm{H}, 4 \mathrm{CH}_{3}\right), 1.46-1.83\left(\mathrm{~m}, 4 \mathrm{H}, 2 \mathrm{CH}_{2}\right)$, $2.13\left(\mathrm{~s}, 4 \mathrm{H}, 2 \mathrm{CH}_{2}\right), 2.26\left(\mathrm{~s}, 4 \mathrm{H}, 2 \mathrm{CH}_{2}\right), 2.56-2.90\left(\mathrm{~m}, 4 \mathrm{H}, 2 \mathrm{CH}_{2}\right) 3.76\left(\mathrm{~s}, 4 \mathrm{H}, 2 \mathrm{CH}_{2}\right), 4.45$ (s, 4H, $\left.2 \mathrm{CH}_{2}\right), 7.10-7.86(\mathrm{~m}, 10 \mathrm{H}) ; \mathrm{MS}: \mathrm{m} / \mathrm{z}, 567\left(\mathrm{MH}^{+}\right)$. Anal. Calc. for $\mathrm{C}_{36} \mathrm{H}_{46} \mathrm{~N}_{4} \mathrm{O}_{2}$ (566.36): C, 76.29; H, 8.18; N, 9.89. Found: C, 76.02; H, 8.21; N, 9.83\%.

\section{3,3'-(1,4-Phenylene)bis(7,7-dimethyl-1-phenyl-1,2,3,4,7,8-hexahydroquinazolin- $5(6 H)$-one $(2 \boldsymbol{h})$}

It was obtained as yellow solid in $89 \%$ yield; mp 126-128 ${ }^{0} \mathrm{C}(\mathrm{MeOH} / \mathrm{EtOAc})$; IR $(\mathrm{KBr})$ : 1507, 1566, $1610 \mathrm{~cm}^{-1} ;{ }^{1} \mathrm{H} \mathrm{NMR}\left(\mathrm{CDCl}_{3}\right): \delta 0.93\left(\mathrm{~s}, 12 \mathrm{H}, 4 \mathrm{CH}_{3}\right), 2.00\left(\mathrm{~s}, 4 \mathrm{H}, 2 \mathrm{CH}_{2}\right), 2.23$ (s, $\left.4 \mathrm{H}, 2 \mathrm{CH}_{2}\right), 4.27\left(\mathrm{~s}, 4 \mathrm{H}, 2 \mathrm{CH}_{2}\right), 4.89\left(\mathrm{~s}, 4 \mathrm{H}, 2 \mathrm{CH}_{2}\right), 6.91-6.96(\mathrm{~m}, 8 \mathrm{H}), 7.27-7.36(\mathrm{~m}$, $6 \mathrm{H}) ;{ }^{13} \mathrm{C} \mathrm{NMR}\left(\mathrm{CDCl}_{3}\right): \delta 28.08,28.52,32.82,41.24,45.96,50.21,52.59,71.21,104.63$, 119.13, 127.53, 127.62, 129.88, 142.84, 157.52, 194.22; MS: m/z, $587\left(\mathrm{MH}^{+}\right)$. Anal. Calc. for $\mathrm{C}_{38} \mathrm{H}_{42} \mathrm{~N}_{4} \mathrm{O}_{2}$ (586.33): C, 77.78; H, 7.21; N, 9.55. Found: C, 77.52; H, 7.18; N, 9.61\%. 


\section{Results and Discussion}

Thus, when a mixture of 3-anilinocyclohex-2-en-1-one (1a), ethylenediamine and formaldehyde $(2: 1: 4)$ in methanol was subjected to MWI, work-up of the reaction mixture followed by chromatographic purification yielded a solid in $85 \%$ yields, which was characterized as 3,3'-(ethane-1,2-diyl)bis(1-phenyl-1,2,3,4,7,8-hexahydroquinazolin-5(6H)one (2a). The reaction was found to be general with other diamines and with corresponding $\mathbf{1 a - b}$ to give the respective $\mathbf{2} \mathbf{b}-\mathbf{h}$ in $\mathbf{7 7 - 9 0 \%}$ overall yields (Table 1 ). These reactions were also carried out under thermal conditions to give the expected products in comparatively lower yields and in addition they took much longer time for completion (Table 2). The structures of the Bis-quinazolines were assigned on the basis of spectral and analytical data. Thus, the infrared spectra of 2a-h showed strong peaks in the range of 1427 to $1619 \mathrm{~cm}^{-1}$ due to highly delocalised double bonds and carbonyl group stretching frequencies of enaminone functionalities. In the ${ }^{1} \mathrm{H}$ NMR spectra of $\mathbf{2 a} \& \mathbf{2 e}$ the $\mathrm{NCH}_{2}$ protons of ethylene chain appeared as singlets at $2.83 \mathrm{ppm}$ and $2.93 \mathrm{ppm}$ respectively whereas the $\mathrm{NCH}_{2}$ protons of propylene chain in $\mathbf{2 b} \& \mathbf{2} \mathbf{f}$ gave triplets in the range of $2.60-2.72 \mathrm{ppm}$. The protons at $\mathrm{C}_{2}$ of propylene chain gave multiplets in the range of 1.59-1.88 ppm. Likewise in $2 \mathbf{c} \& \mathbf{2 g}$ the protons at $\mathrm{C}_{1} \& \mathrm{C}_{2}$ of butylene chains gave multiplets in the range of 2.56-2.90 and 1.46$1.88 \mathrm{ppm}$ respectively. The protons at $\mathrm{C}_{2}$ and $\mathrm{C}_{4}$ of quinazoline ring resonated in the range of 4.24-4.99 and 3.64-4.56 ppm respectively. In $\mathbf{2 a - d}$ the $\mathrm{C}_{8}$ protons of the parent ring appeared as triplets between $2.10 \& 2.30 \mathrm{ppm}$ but in $\mathbf{2 e - h}$ they appeared as singlets between $1.99 \& 2.10 \mathrm{ppm}$. The $\mathrm{C}_{6}$ protons of the parent ring in 2a-d appeared as triplets between $2.31 \& 2.60 \mathrm{ppm}$ but in $\mathbf{2 e - h}$ they appeared as singlets between $2.13 \& 2.26 \mathrm{ppm}$. The $\mathrm{C}_{7}$ protons of the ring in 2a-d resonated giving multiplets in the range of $1.58-2.06 \mathrm{ppm}$. The two-methyl protons at $\mathrm{C}_{7}$ in $\mathbf{2 e - h}$ gave singlets between $0.90 \& 1.03 \mathrm{ppm}$. The aromatic protons resonated in their usual range of $6.91-7.93 \mathrm{ppm}$. A plausible mechanism for the formation of $\mathbf{2}$ from the cyclic enaminones $\mathbf{1}$ is worked out (Scheme 2).
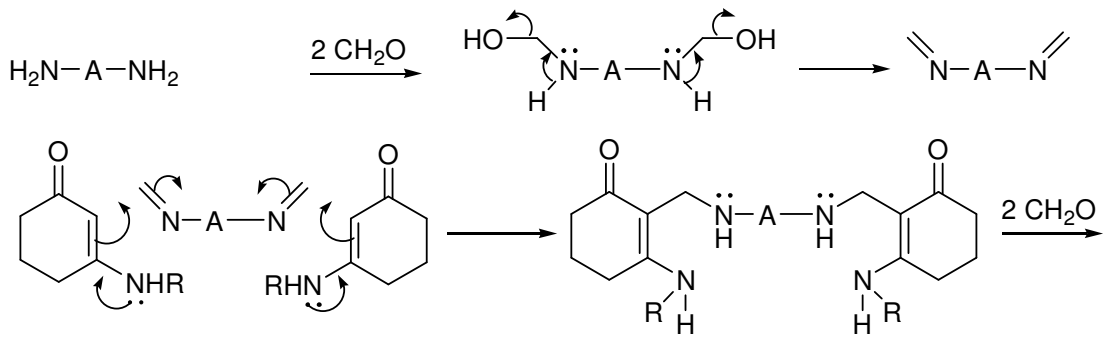

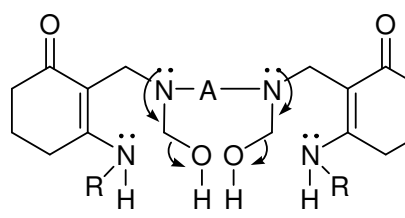

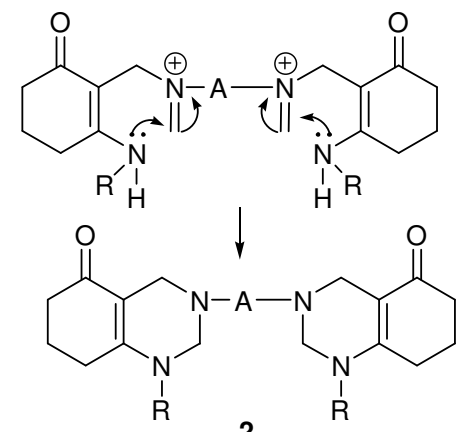

Scheme 2. 
Table 1. Synthesis of bis-quinazolinones (2a-h)

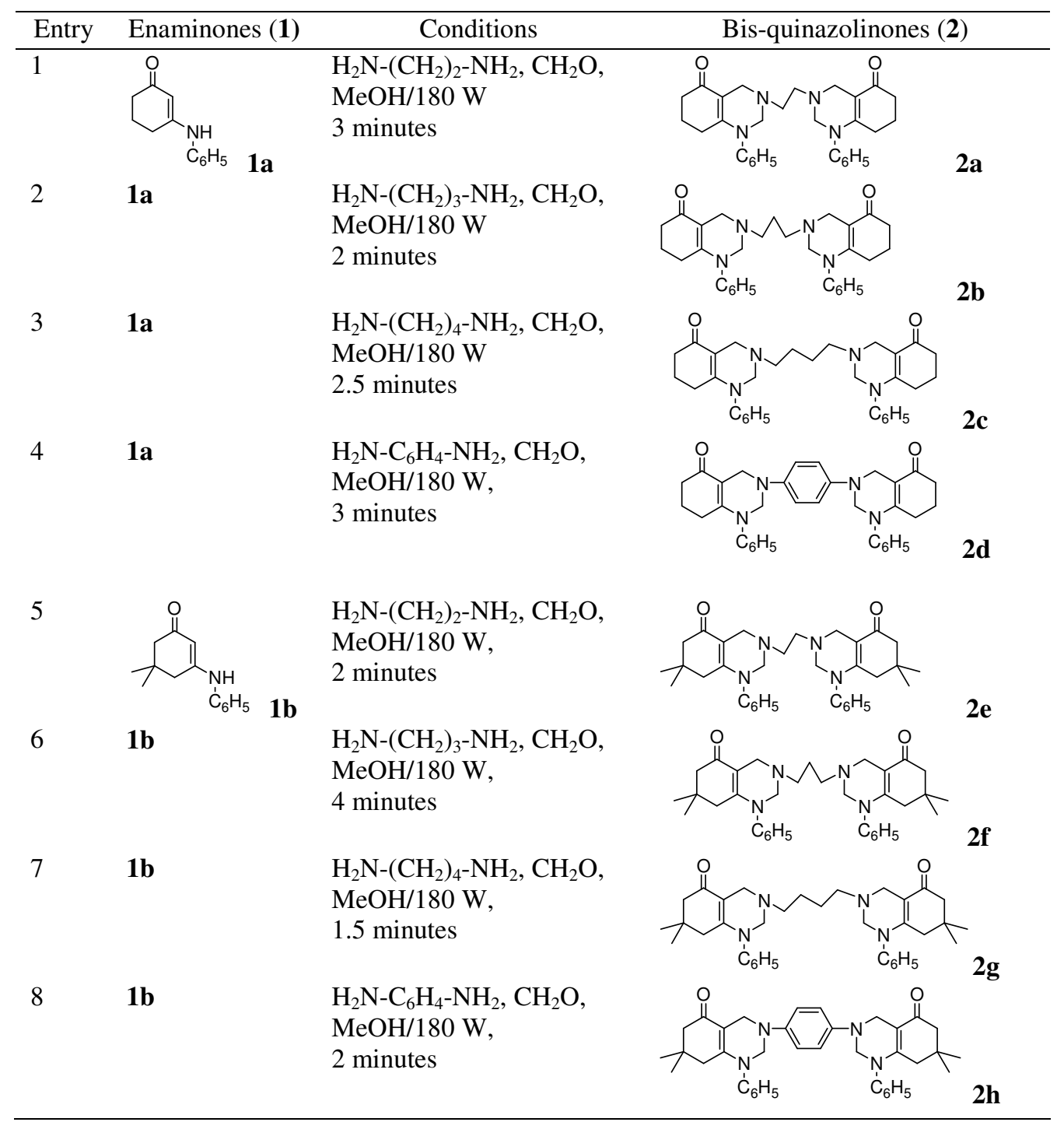

Table 2. Comparison of microwave irradiation and thermal condition results.

\begin{tabular}{ccccc}
\hline \multirow{2}{*}{ Compound } & \multicolumn{2}{c}{ Microwave Irradiation $(180 \mathrm{~W})$} & \multicolumn{2}{c}{ Thermal (Reflux) } \\
& Time, $\min$ & Yield, $\%$ & Time, & Yield, \% \\
\hline $\mathbf{2 a}$ & 3 & 85 & 27 & 58 \\
$\mathbf{2 b}$ & 2 & 76 & 22 & 70 \\
$\mathbf{2 c}$ & 2.5 & 77 & 24 & 65 \\
$\mathbf{2 d}$ & 3 & 79 & 24 & 71 \\
$\mathbf{2 e}$ & 2 & 87 & 22 & 88 \\
$\mathbf{2 f}$ & 4 & 75 & 23 & 68 \\
$\mathbf{2 g}$ & 1.5 & 90 & 8 & 59 \\
$\mathbf{2 h}$ & 2 & 89 & 20 & 55 \\
\hline
\end{tabular}




\section{Acknowledgements}

The authors wish to thank the Principal, Rev. Fr. Ioannis Warpakma, SDB for the facilities and Rev. Fr. Stephen Mavely, SDB and Rev. Fr. Joseph Nellanatt, SDB for their encouragement during the course of this investigation. The financial support from ICARNATP-PIU and UGC-New Delhi is gratefully acknowledged. Authors (KC \& MCD) thank ICAR for Senior Research Fellowships. Thanks are also due to the Heads of RSIC-CDRI (Lucknow) and RSIC-NEHU (Shillong) for recording spectra.

\section{References}

1. Taylor E C and Patel M, J Heterocyclic Chem., 1991, 28, 1857.

2. Coates W J, Comprehensive Heterocyclic Chemistry II, Vol 6, Edited by Boulton A J, Pergamon, Oxford, 1996, 225-231.

3. Elslager E F, Hess C, Johnson J, Ortwine D, Chu V and Werbel L M, J Med Chem., 1981, 24, 127.

4. Rivero I A, Espinoza K and Somanathan R, Molecules, 2004, 9, 609 and references cited therein.

5. Sarac S, Yarim M, Ertan M, Kilic F S and Erol K, Arzneim Forsch Drug Res., 2002, 52, 27.

6. Yarim M, Sarac S, Kilic F S and Erol K, Il Farmaco, 2003, 58, 17.

7. Kidwai M, Saxena S, Khan M K R and Thukral S S, Eur J Med Chem., 2005, 40, 816.

8. Kantevari M, Bantu R and Nagarapu L, ARKIVOC, 2006, 16, 136.

9. Hamama W S, Hammouda M and Afsah E M, Z. Naturforsch, 1988, 43B, 483; Chem. Abstr., 1988, 109, 170359k.

10. Chanda K, Dutta M C and Vishwakarma J N, Indian J Chem., 2006, 45B, 1076.

11. Vishwakarma J N, Mofizuddin M, Ila H and Junjappa H, J Heterocyclic Chem., 1988, 25, 1387.

12. Karim E, Kishore K and Vishwakarma J N, J Heterocyclic Chem., 2003, 40, 901.

13. Chanda K, Dutta M C, Karim E and Vishwakarma J N, J Heterocyclic Chem., 2004, 41, 627.

14. Dutta M C, Chanda K and Vishwakarma J N, J Heterocyclic Chem., 2005, 42, 121.

15. Chanda K, Dutta M C and Vishwakarma J N, Indian J Chem., 2004, 43B, 2475. 


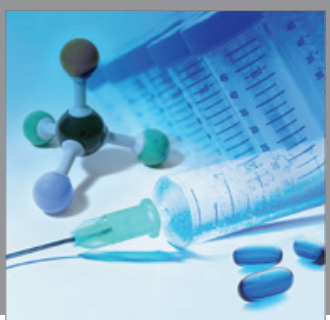

International Journal of

Medicinal Chemistry

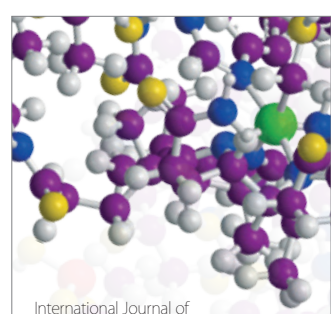

Carbohydrate Chemistry

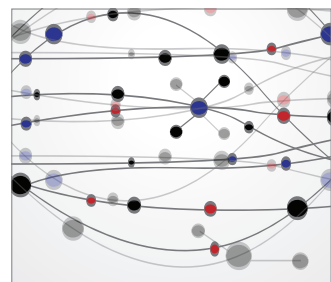

The Scientific World Journal
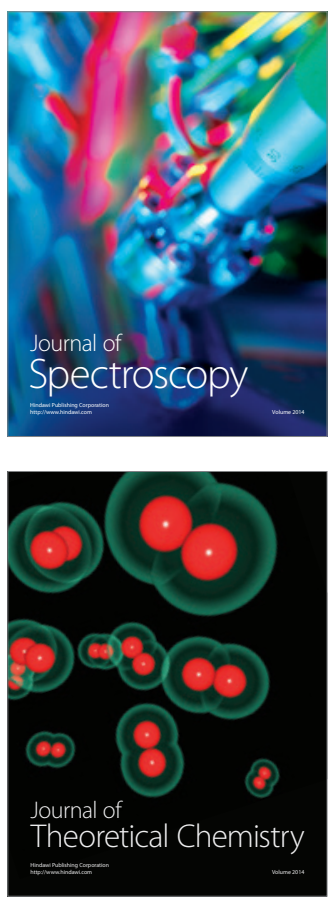
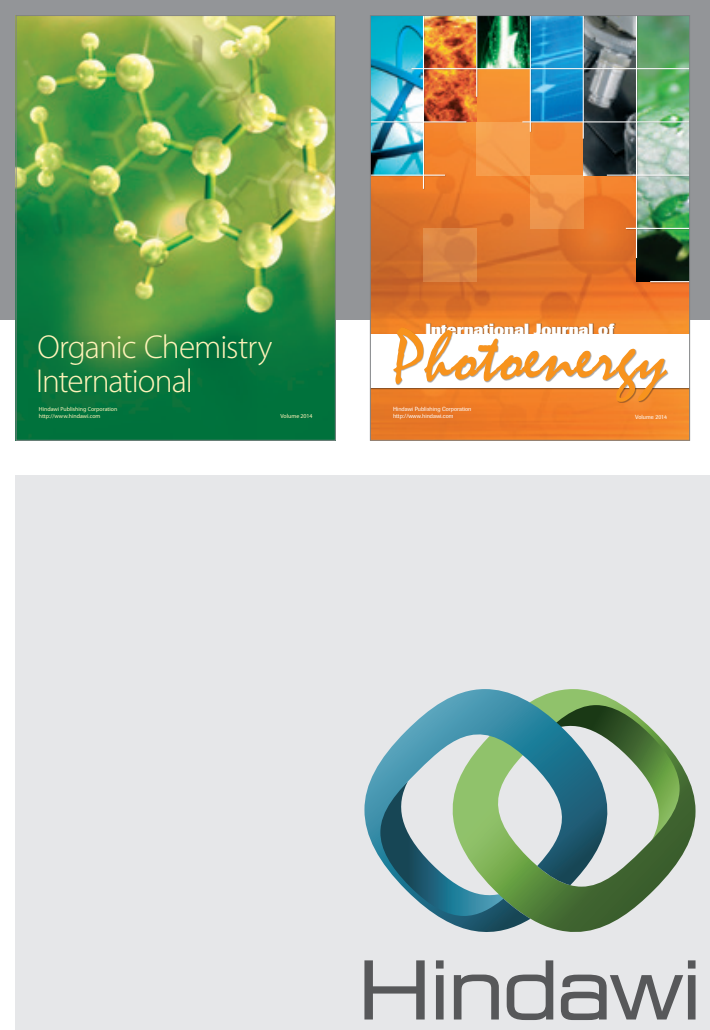

Submit your manuscripts at

http://www.hindawi.com
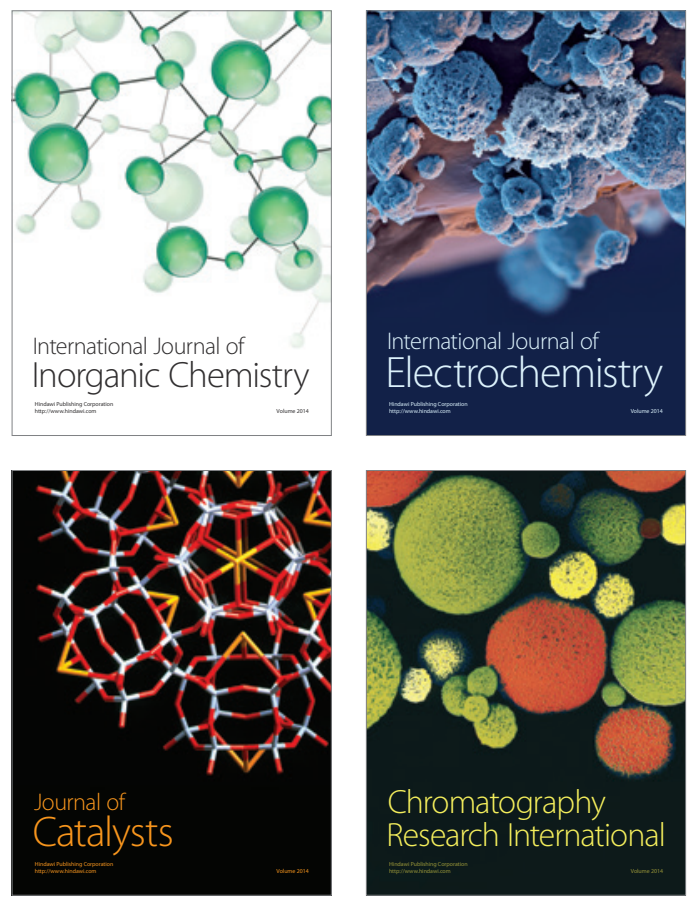
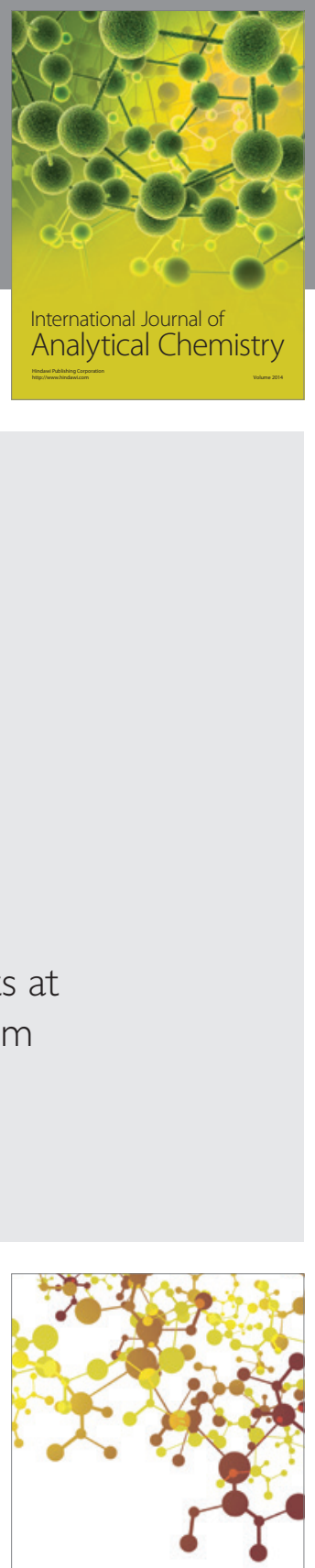

Journal of

Applied Chemistry
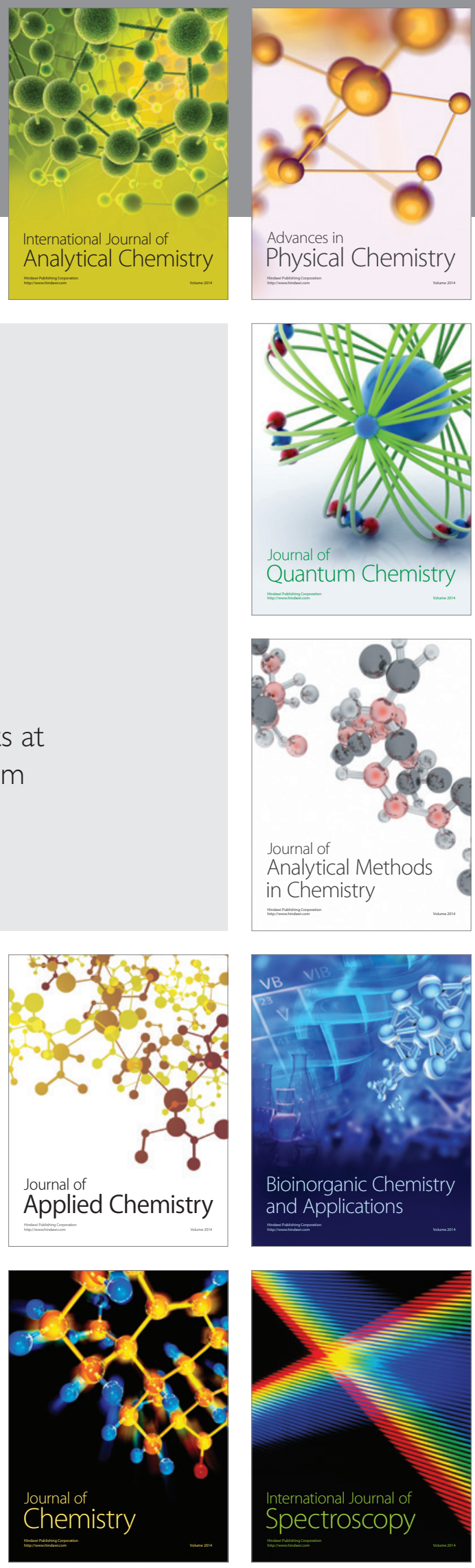